\title{
POSTER
}

\section{Amylose systémique à manifestation buccale : à propos d'un cas}

\section{Dimmock M, Cros A, Laurencin S, Benat G, Cousty S}

L'amylose AL, également appelée amylose primitive ou amyloïdose est une maladie rare. Elle se caractérise par la présence de dépôts amyloïdes à chaines légères d'immunoglobulines au niveau des tissus en feuillet beta plissé. Le diagnostic positif est histologique, il révèle une coloration rouge congo positive qui signe la présence de protéines amyloïdes. Les localisations préférentielles de la maladie sont : rénales, cardiaques, hépatiques, cutanées et nerveuses. La localisation cervico-bucco-faciale est rare, elle associée le plus souvent à une atteinte systémique et plus rarement à une forme localisée.

Une femme de 56 ans, d'origine afghane, consulte pour des dysphagies et des difficultés d'élocution récentes. La patiente présente une sclérodermie systémique et une amylose systémique diagnostiquée 10 mois auparavant par une macroglossie avec une biopsie de la face interne de la joue. Elle manifeste une atteinte rénale, hépatique, pulmonaire, nerveuse et thyroïdienne de sa maladie. Son traitement actuel est une chimiothérapie combinant velcade, endoxan et dexamethasone. L'examen clinique retrouve une ouverture buccale limitée, en rapport avec des dépôts amyloïdes granuleux au niveau de la lèvre inférieure et la face interne des joues. Une macroglossie indolore, avec indentations sur l'ensemble du pourtour lingual est notée, elle est cependant en amélioration depuis l'introduction de la thérapeutique systémique.

II existe une hyposialie marquée, sans complication parodontale. L'examen des aires cervicales est difficile mais ne rapporte pas d'adénopathie en première intention.

L'examen fonctionnel retrouve des difficultés à la déglutition et à la mobilité linguale. La phonation est également perturbée.

Au travers de ce cas clinique, il est rappelé les différentes manifestations buccales de l'amylose AL. Ces manifestations peuvent être inaugurales de l'atteinte systémique. Actuellement, il n'existe pas de thérapeutique locale spécifique. La régression des manifestations buccales repose essentiellement sur la chimiothérapie générale. Le chirurgien oral peut intervenir au niveau diagnostique (biopsie de la muqueuse buccale, biopsie des glandes salivaires accessoires, mettent en évidence l'amylose) mais également au niveau de la thérapeutique, dans la gestion des complications locales de l'amylose et des traitements systémiques de celle-ci. Le rôle d'accompagnement au niveau « oral » de ces patients est primordial et nécessite une équipe multidisciplinaire comprenant chirurgiens oraux, ORL et orthophonistes.

Références

1) Localized Amyloidosis of the tongue : A review Kristen S et al. AM J Otolaryngol, 2004; 25 : 186-189.

2) Localized amyloidosis of the upper gingiva : a case report Bucci T et al. J Med Case Rep. 2014; 8 : 198.

3) Unsual presentation of oral amyloidosis : William P et al. Contemp Clin Dent, 2015; 6(Suppl 1): S282-S284.

This is an Open Access article distributed under the terms of the Creative Commons Attribution License 4.0, which permits unrestricted use, distribution, and reproduction in any medium, provided the original work is properly cited. 